\section{Wilson's Disease}

Anna DePold Hohler ${ }^{1}$ and Marcus Ponce de Leon ${ }^{2}$ ${ }^{1}$ Boston University Medical Center, Boston, MA, USA

${ }^{2}$ Madigan Army Medical Center, Tacoma, WA, USA

\section{Synonyms}

Hepatolenticular degeneration

\section{Definition}

Wilson's disease is a disorder of copper metabolism absence or dysfunction of a copper transporting P-type ATPase. The chromosome 13 autosomal recessive mutation causes a defect in copper metabolism, causing its buildup in the liver, brain, and eye.

\section{Current Knowledge}

Numerous cognitive, psychiatric, and movement disorders may be seen with this disease. Wilson's disease neurologic manifestations include tremor, dystonia, dysmetria, dysrhythmia, ataxia, and dysarthria. Tremor is typically a proximal or "wing beating tremor." Testing for Wilson's disease involves blood and urine testing of liver function tests, ceruloplasmin, and serum and urine copper. Liver function abnormalities in the face of low ceruloplasmin and high urinary copper levels should prompt a diagnosis of Wilson's disease. Slit-lamp examination may reveal KayserFleischer rings. MRI often shows abnormal T2 signal in the basal ganglia. The typical patient presents in early or middle adulthood with hepatic, psychiatric, and/or neurologic symptoms.

\section{Cross-References}

Dystonia

\section{References and Readings}

Pfeiffer, R. F. (2004). Wilson's disease. In R. L. Watts \& W. C. Koller (Eds.), Movement disorders (2nd ed., pp. 779-798). New York: McGraw-Hill. 